\title{
Dendrite Growth Model at Bonded (001) GaAs Interface
}

\author{
Yew Chung Sermon Wu, A.Panimaya Selvi Isabel \\ Department of Materials Science and Engineering, National Chiao Tung University, Hsinchu, Taiwan
}

\begin{abstract}
A periodic GaAs wafer-bonded structure was used for nonlinear optical device. Results showed voids present at the bonded GaAs twin boundary. These voids were caused by natural topographical irregularities and surface contamination. Most contamination could be eliminated by sulfur passivation treatments. However, natural irregularities are inevitable. After bonding, two kinds of crystallites were formed at the bonded (001) interfacial voids: diamond-shaped and dendritic geometries. This paper discussed the dendrite growth models within the interfacial void regions.
\end{abstract}

Keywords: nonlinear optical materials, quasi-phase-matched second-harmonic generation, GaAs wafer bonding

\section{Introduction}

A periodic structure of bonded GaAs wafers was used for quasi-phase-matched second-harmonic generation. In this structure, each wafer had to be rotated $180^{\circ}$, thus creating a twin boundary at the interface. Voids were always found at the bonded interface. [1-3] These voids were caused by natural topographical irregularities and surface contamination (including particles, solvent residues, and trapped gases) between the wafers. Most surface contamination could be eliminated using sulfur passivation and subsequent treatments. $[1,4]$ However, natural irregularities are inevitable because atomically smooth wafers are not available. After bonding, two kinds of bright features (crystallites) were formed at the bonded (001) interfacial voids: diamond-shaped and dendritic geometries. This study examined the dendrite growth kinetics at the (001) GaAs bonded interface.

\section{Experimental}

Undoped semi-insulating (001) GaAs wafers were used. They were diced into $9 \mathrm{x} 9 \mathrm{~mm}^{2}$ samples, and then cleaned in a clean room. GaAs wafer was rotated by $180^{\circ}$ to create a periodic bonded structure. [1-4] Bonding occurred in the temperature range of $850^{\circ} \mathrm{C}-950^{\circ} \mathrm{C}$, and created a twin boundary at the interface.

\section{Results And Discussion}

Figure 1 shows the IR transmission microscopy-observed crystal features after bonding. As can be seen, when the void depth (formed by surface irregularities) was $\geq 200 \mathrm{~nm}$, most of the bright features (bonded regions) were diamond-shaped (Fig. 1(a)). On the other hand, when the void depth was small $(\mathrm{h} \leq 70 \mathrm{~nm})$, the bright features were mainly of dendritic geometries (Fig. 1(b)). [2, 3] Differences in crystal features can be attributed to the driving force, which is a function of void depth and can be estimated by the Gibbs-Thomson equation as follows.

$$
\Delta G_{T} \approx-2 k^{\prime} \frac{\gamma}{h}
$$

where $\gamma$ is the free energy of the flat surface, $h$ is the depth of the void, and $k^{\prime}$ is the geometry constant. When $h$ was $\leq 70 \mathrm{~nm}$, the driving force was larger. The growth of GaAs crystallites (bright features) results from growth rate anisotropy and dendrites grow quickly along the fast-growing $\langle 110\rangle$ direction. The dendritic cap is bounded by fast-growing $\{111\}$ planes as shown in Fig. 1(c). The dendrite growth model is analyzed and discussed in the following section.

As shown in Fig. 2(a), the growth of dendrites/crystallites during the bonding/healing process is divided into three stages: evaporation from unbonded (001) wafer surface, mass transport, and deposition on the exposed (bonded) surface. The geometry and growth rates of dendrites are controlled by the slowest of the three stages; that is, the deposition on the exposed bonded surface. [3]

The related energies are illustrated in Figs. 2(b) and 2(c). The driving force behind evaporation is given by

$$
\Delta G_{\text {evap }}=G_{001 F}-G_{001},
$$

where $G_{001}$ is the free energy of the unbonded (001) surface, and $G_{001 F}$ is the free energy of the "species" in front of the (001) surfaces. The driving force for mass transport is given by

$$
\Delta G_{\text {tran }}=G_{\text {FacetF }}-G_{001 F},
$$


where $G_{\text {FacetF }}$ is the free energy of species in front of the bonded surface. The driving force behind deposition is given by

$$
\Delta G_{\text {dep }}=G_{\text {Facet }}-G_{\text {FacetF }},
$$

where $G_{\text {Facet }}$ is the free energy of the bonded surface. The total driving force is thus given by

$$
\begin{aligned}
\Delta G_{T} & =\Delta G_{\text {evap }}+\Delta G_{\text {tran }}+\Delta G_{\text {dep }} \\
& =G_{\text {Facet }}-G_{001}=-2 k^{\prime} \frac{\gamma}{h} .
\end{aligned}
$$

The mathematical treatment of the growth stages is discussed below.

\section{A. Evaporation from unbonded (001) surface}

The evaporation of Ga and As atoms involves breaking GaAs bonds, which incurs an energy $\operatorname{cost}\left(\Delta G_{A}^{* \text { evap }}\right)$ as shown in Fig. 3(a). As a result, the evaporation rate $V_{\text {evap }}$ is proportional to

Where

$$
\begin{gathered}
V_{\text {evap }} \propto\left[\exp \left(-\Delta G_{A}^{* \text { evap }} / R T\right)-\exp \left(-\left(\Delta G_{A}^{* \text { evap }}+\Delta G_{K}^{\text {evap }}\right) / R T\right)\right] \\
=\exp \left(-\Delta G_{A}^{* \text { evap }} / R T\right)\left[1-\exp \left(-\Delta G_{K}^{\text {evap }} / R T\right)\right]
\end{gathered}
$$

$$
\begin{gathered}
\Delta G_{A}^{\text {*evap }}=G_{001}^{*}-G_{001}, \\
\begin{array}{c}
\Delta G_{K}^{\text {evap }}=G_{001}-G_{001 F} \\
=-\Delta G_{\text {evap }}
\end{array}
\end{gathered}
$$

where $G_{001}^{*}$ is the free energy of the activated state for this evaporation and $\Delta G_{A}^{* \text { evap }}$ is the effective energy barrier for evaporation. Evaporation from unbonded (001) wafer surface is not the slowest or limiting stage.[3]

\section{B. Mass Transport}

As shown in Fig. 2(a), Ga and As atoms must transport either through the GaAs lattice, along the unbonded GaAs surface and/or through the gas phase. At temperatures above $850^{\circ} \mathrm{C}$, the As equilibrium pressure is high (> 60 torr). With the As transport rate through the gas phase much higher than that through other channels, gas-phase transport must be the dominant path. On the other hand, the vapor pressure of Ga over GaAs is markedly small and can thus be neglected. [5] The diffusion coefficients of Ga follow the relationship below. [6-8]

$$
\begin{gathered}
D_{G a}^{l}=0.1 e^{-3.2 / k T} \mathrm{~cm}^{2} \mathrm{~s}^{-1} \\
D_{G a}^{v}=2.1 \times 10^{-3} e^{-2.1 / k T} \mathrm{~cm}^{2} s^{-1} \\
D_{G a}^{s}=1.6 \times 10^{-2} e^{-1.1 / k T} \mathrm{~cm}^{2} s^{-1}
\end{gathered}
$$

where $D_{G a}^{l}$ is the lattice diffusion coefficient of Ga atoms, $D_{G a}^{v}$ is the diffusion coefficient of Ga vacancies, and $D_{G a}^{s}$ is the surface diffusion coefficient of $\mathrm{Ga}$ atoms.

At temperatures ranging between $850^{\circ} \mathrm{C}$ and $950^{\circ} \mathrm{C}$, the diffusion coefficient of $\mathrm{Ga}, D_{G a}^{*}$ (including $D_{G a}^{l}, D_{G a}^{v}$, and $D_{G a}^{s}$ ) is less than $<10^{-6} \mathrm{~cm}^{2} \mathrm{~s}^{-1} . \quad D_{G a}^{*}$ is much lower than that of the As vapor transport rate $\left(D_{A s} \sim 10^{2} \mathrm{~cm}^{2} \mathrm{~s}^{-1}\right)$; [9] therefore, the mass transport of GaAs is limited by Ga diffusion.

The mass transport rate can be modeled by the following one-dimensional diffusion equation with the reference point at the reentrant corner of the twin boundary

$$
V_{\text {tran }} \propto D_{G a}^{*} \frac{\partial \Delta G_{\text {tran }}(x, t)}{\partial x}=D_{G a}^{*} \frac{\partial G_{\text {tran }}(x, t)}{\partial x}
$$

where $x$ is the distance from the reentrant corner and $G_{t r a n}(x, t)$ is the free energy of Ga along the free surface of void. 
The boundary condition is

$$
G_{\text {tran }}(0, t)=G_{\text {FacetF }} \quad G_{\text {tran }}(\infty, t)=G_{001 F}
$$

By analyzing equations (1) and (2), the free energy distribution of "transport mass" looks more or less like the curve shown in Fig. 4.

\section{Deposition on bonded surface}

The deposition stage involves two steps: nucleation of new surface layer and surface layer motion, as shown in Fig. 2(d). Previous research has found that surface layer nucleation is the slower limiting step.[3] In other words, nucleation of a new layer is the slowest step in the growth of dendrites.

Surface layer nucleation is described by a partial pill-box island at the reentrant corner.[10] When the rate of layer motion is infinitely rapid compared with that of formation of new surface layer, the dendrite growth rate is proportional to

$$
V \propto \exp \left(-\Delta G_{A}^{* d e p} / R T\right)\left[1-\exp \left(-\Delta G_{K}^{d e p} / R T\right)\right\rfloor
$$

where

$$
\begin{aligned}
\Delta G_{A}^{* d e p} & =G_{\text {Facet }}^{*}-G_{\text {FacetF }} \\
\Delta G_{K}^{\text {dep }} & =G_{\text {FacetF }}-G_{\text {Facet }} \\
& =-\Delta G_{\text {dep }}
\end{aligned}
$$

where $G_{F a c e t}^{*}$ is the free energy of the activated state, and $\Delta G_{A}^{*}$ dep is the effective energy barrier to the formation of a new layer as shown in Fig. 3(b). If the layer motion rate is not infinitely rapid, then Eq. (1) needs to be multiplied by a factor $<1$. [11]

\section{Growth of dendrites}

The relative mass transport driving force $\left(\Delta G_{\text {tran }}\right)$, deposition driving force $\left(\Delta G_{d e p}\right)$, and the free energy of $\mathrm{Ga}\left(G_{\text {tran }}(x, t)\right)$ in front of the reentrant corner ("dendrite cap") are shown in Figs. 4(a) and (b). A protrusion $\Delta x$ (Fig. 4(c)) which sticks out in front of the reentrant corner will have an extra driving force $\Delta G_{d e p}^{e x}$ (Figs. 4(a) and (b)) for the deposition of atoms. The extra driving force also decreases with void depth.

For shallow voids (Fig. 4(a)), a protrusion $\Delta x$ sticking out from the reentrant corner will have a huge $\Delta G_{d e p}^{e x}$.

This huge driving force first causes the nucleation of new layers on the reentrant corner, as shown in Fig. 2(d). These layers then grow rapidly along the surface plane in a layer-by-layer process, resulting in the forward motion (growth) of crystallites. Its growth velocity is increased relative to the surrounding surface, which is in contact with Ga atoms at a higher temperature. [12] The difference in growth velocity can be attributed to the release of latent heat associated with the development of each protrusion. This heat raises the temperature of the $\mathrm{Ga}$ atoms adjacent to the protrusion and retards the nucleation of new layers in the immediate vicinity of a given protrusion. In particular, where fast-growing planes such as the $\{111\}$ are present, the crystallite growth rate will increase exponentially with the driving force, thus resulting in dendrite growth in the $<110\rangle$ direction. On the other hand, as shown in Fig. 4(b), the driving force at deep voids is too small to cause dendrite growth.

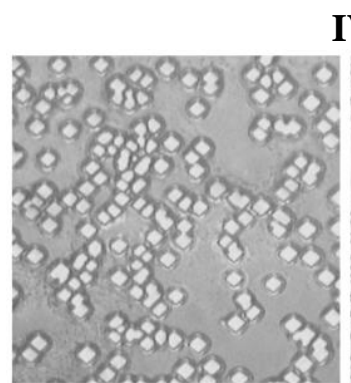

(a)

\section{Figure Captions}

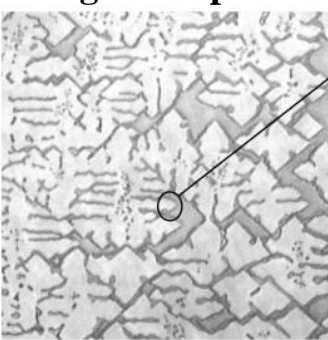

(b)

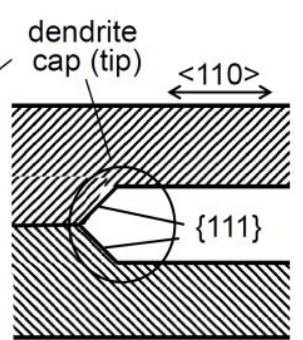

(c)

Fig. 1. SEM images of two kinds of crystal features formed at bonded (001) interfacial voids: (a) diamond (void depth $\geq 200 \mathrm{~nm}$ ) and (b) dendrite (void depth $\leq 70 \mathrm{~nm}$ ). (c) is cross-sectional illustration of dendrite cap. 


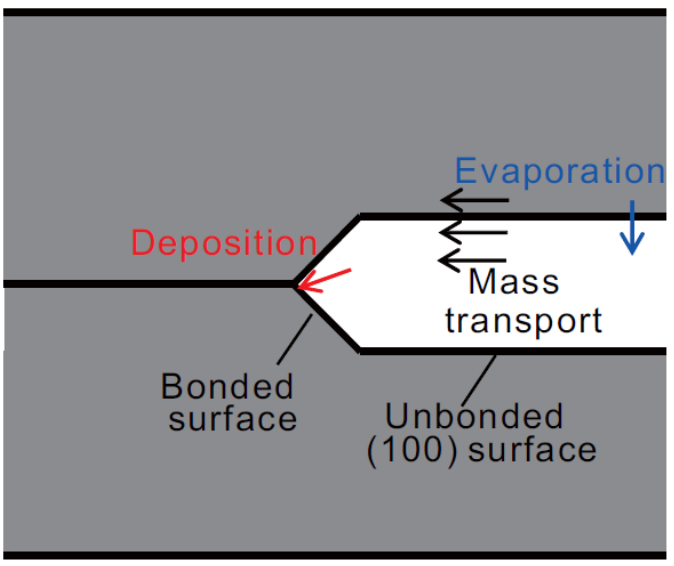

(a)

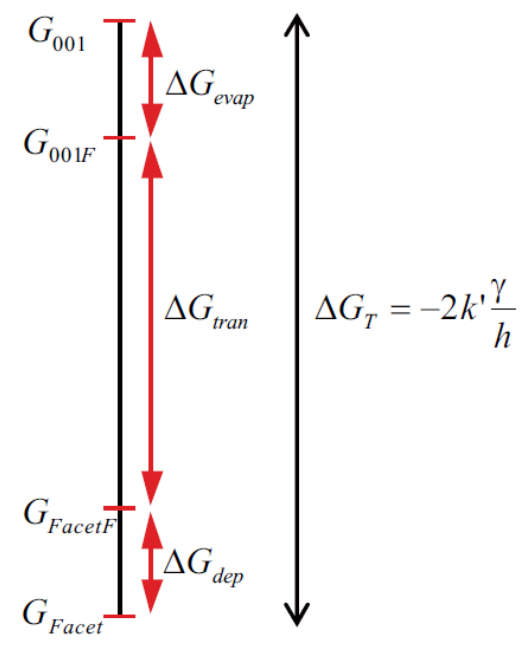

(b)

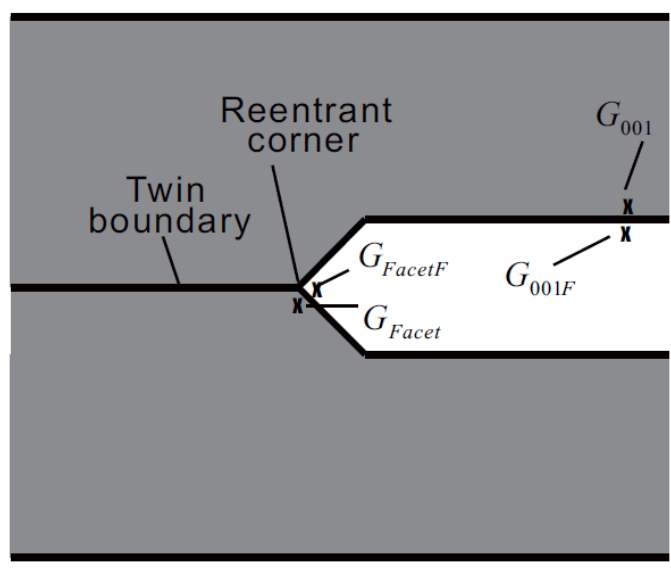

(c)

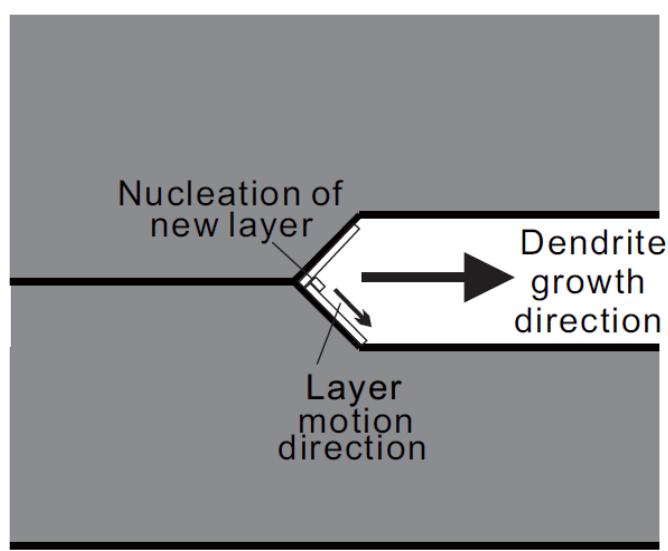

(d)

Fig. 2 Schematic illustration of dendrite growth stages and related free energies. (a) shows the three growth stages: evaporation, mass transport and deposition. (b) and (c) illustrates the related free energies. (d) shows the two deposition steps: surface layer nucleation and and layer motion.

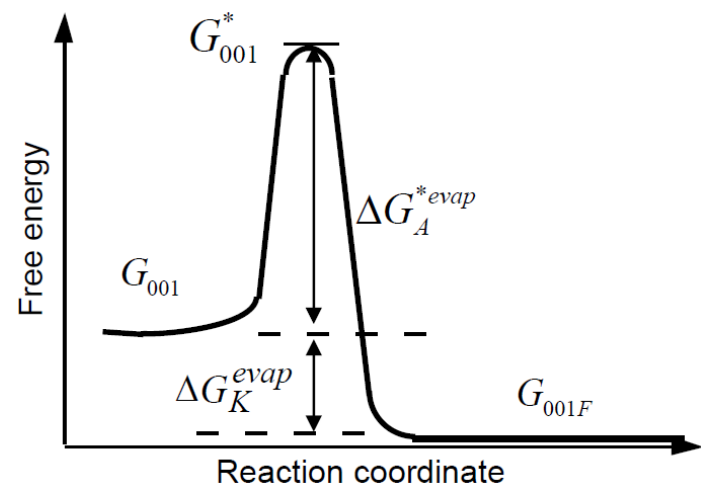

(a)

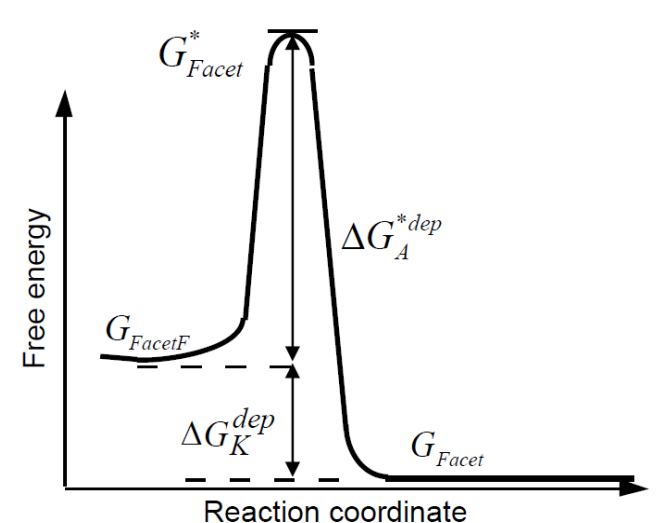

(b)

Fig. 3. Key free energies involved in (a) evaporation from unbonded (001) surface, and (b) deposition on bonded surface. 


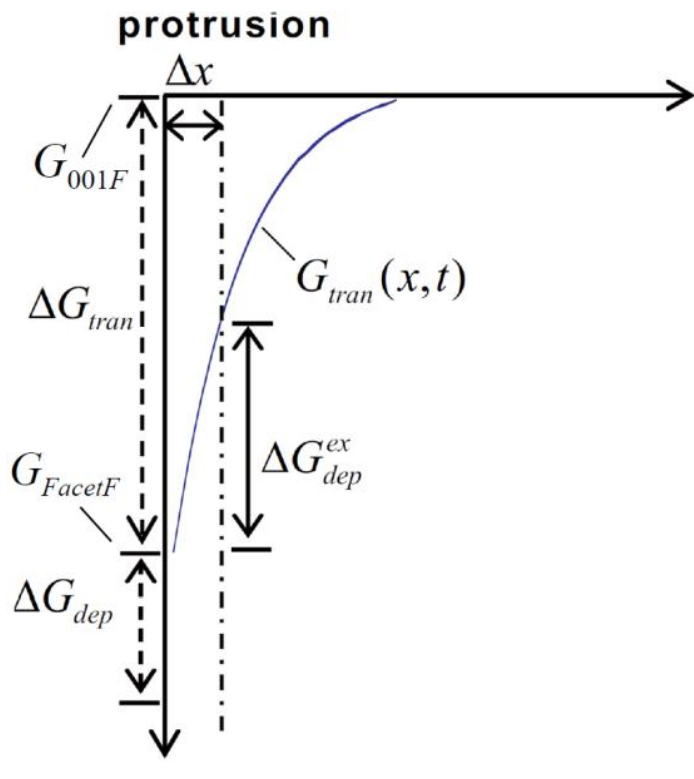

(a)

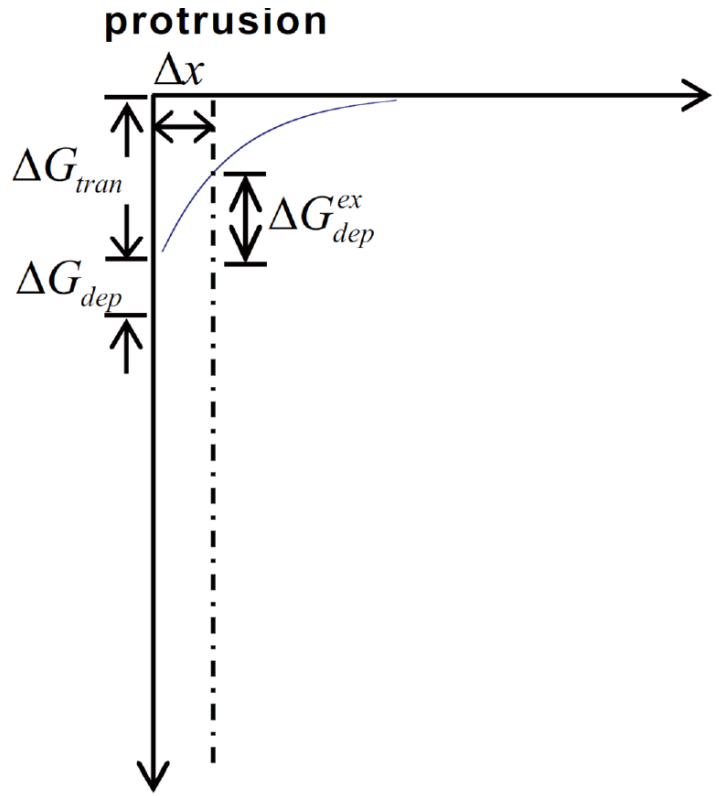

(b)

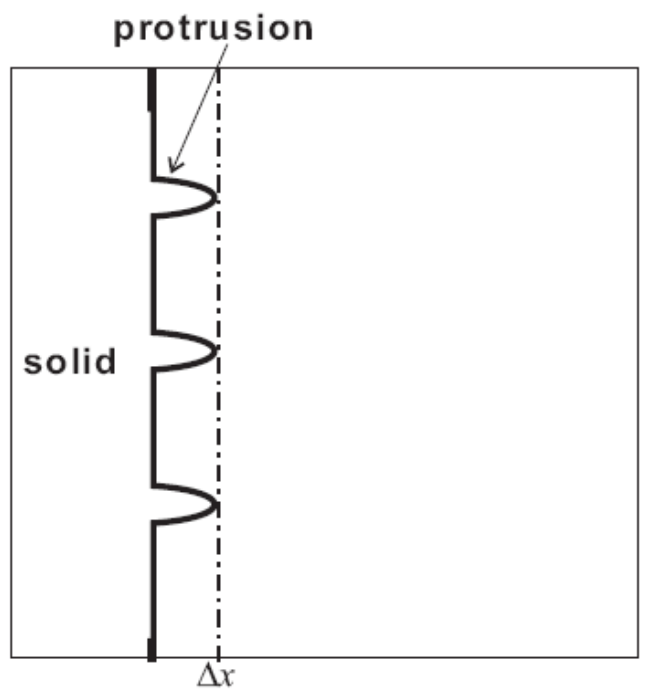

(c)

Fig. 4. Key free energies involved in dendrite growth with protrusion in front of reentrant corner for (a) shallow and (b) deep voids. (c) shows the schematic illustration of protrusions.

\section{Conclusion}

A periodic structure of bonded GaAs wafers was used for quasi-phase-matched second-harmonic generation. Interfacial voids were found at the (001) GaAs bonded twin boundary. These voids were caused by natural topographical irregularities on the wafer surface. Two kinds of crystallites were formed at these voids: diamond-shaped and dendritic geometries. When void depth was $\leq 70 \mathrm{~nm}$, the growth of crystallites inside the voids results from growth rate anisotropy and dendrites grow quickly along the fast-growing $\langle 110\rangle$ direction.

The growth of dendrites involves three stages: evaporation from the unbonded (001) wafer surface, mass transport, and deposition on the bonded surface, which is the slowest/limiting stage. There are two steps in the deposition stage: surface layer nucleation and surface layer motion. Surface layer nucleation is the slower limiting step. For shallow voids, a protrusion sticking out from the reentrant corner will have a huge extra driving force. The crystallite growth rate will increase exponentially with this driving force, resulting in dendrite growth in the $\langle 110>$ direction. 


\section{Acknowledgments}

This project was funded by Sino American Silicon Products Incorporation and the National Science Council of the Republic of China under Grant No. MOST104-2622-E-009-011-CC3. Technical support from the National Nano Device Laboratory, Center for Nano Science and Technology, Nano Facility Center and Semiconductor Laser Technology Laboratory of the National Chiao Tung University is also gratefully acknowledged.

\section{References}

[1]. M. J. Jackson, B. L. Jackson, and M. S. Goorsky, Reduction of the potential energy barrier and resistance at wafer-bonded n-GaAs/n-GaAs interfaces by sulphur passivation J. Appl. Phys., 110, 104903 (2011)

[2]. Y. S. Wu, and P. C. Liu, R. S. Feigelson and R. K. Route, High-temperature healing of interfacial voids in GaAs wafer bonding J. Appl. Phys., 91, 1973 (2002).

[3]. Y. S. Wu and G. Z. Hu, Healing kinetics of interfacial voids in GaAs wafer bonding Appl. Phys. Lett. 81, 1429(2002).

[4]. R. S. Besserand and C. R. Helms, Comparison of surface properties of sodium sulfide and ammonium sulfide passivation of GaAs $J$. Appl. Phys. 65, 4306 (1989).

[5]. J.R. arthur, Vapor pressures and phase equilibria in the GaAs system J. Phys.Chem.solids 28, 2257 (1967).

[6]. S. K. Ghandhi, VLSI Fabrication Principles - Silicon and Gallium Arsenide, 2nd ed. p.197, J. Wiley, New York (1994).

[7]. S. Y. Chiang, and G. L. Pearson, Properties of vacancy defects in GaAs single crystals J. Appl. Phys., 46, 2986 (1975)

[8]. T. Nishinaga and K. Cho, Theoretical Study of Mode Transition between 2d-Nucleation and Step Flow in MBE Growth of GaAs, Jpn. J. Appl. Phys. 27, L12 (1988).

[9]. M. Ohring, The Materials Science of Thin Films, 2nd ed. p.296, Academic Press, San Diego (2002).

[10]. W. A. Tiller, The Science of Crystallization Microscopic Interfacial Phenomena, p.76, Cambridge University, Cambridge (1991).

[11]. W. A. Tiller, The Science of Crystallization Microscopic Interfacial Phenomena, p.76, Cambridge University, Cambridge (1991).

[12]. Robert E., Reed-Hill and R. Abbaschian, Physical Metallurgy Principles 3rd ed. (Thomson, Boston, 1992) p445. 